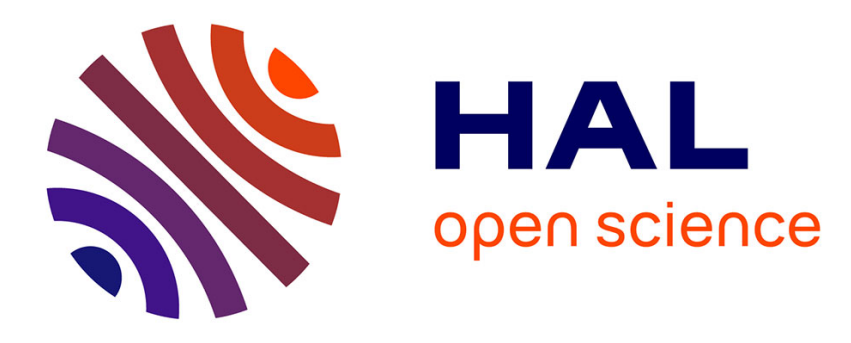

\title{
New Finite-Time and Fast Converging Observers with a Single Delay
}

\author{
Frédéric Mazenc, Michael Malisoff
}

\section{To cite this version:}

Frédéric Mazenc, Michael Malisoff. New Finite-Time and Fast Converging Observers with a Single Delay. Systems and Control Letters, 2022, 6, pp.1561-1566. 10.1109/LCSYS.2021.3123688 . hal03430682

\section{HAL Id: hal-03430682 \\ https://hal.inria.fr/hal-03430682}

Submitted on 16 Nov 2021

HAL is a multi-disciplinary open access archive for the deposit and dissemination of scientific research documents, whether they are published or not. The documents may come from teaching and research institutions in France or abroad, or from public or private research centers.
L'archive ouverte pluridisciplinaire HAL, est destinée au dépôt et à la diffusion de documents scientifiques de niveau recherche, publiés ou non, émanant des établissements d'enseignement et de recherche français ou étrangers, des laboratoires publics ou privés. 


\title{
New Finite-Time and Fast Converging Observers with a Single Delay
}

\author{
Frédéric Mazenc and Michael Malisoff
}

\begin{abstract}
We provide new reduced order observer designs for a key class of nonlinear dynamics. When continuous output measurements are available, we prove that our observers converge in a fixed finite time in the absence of perturbations, and we prove a robustness result under uncertainties in the output measurements and in the dynamics, which bounds the observation error in terms of bounds on the uncertainties. The observers contain a dynamic extension with only one pointwise delay, and they use the observability Gramian to eliminate an invertibility condition that was present in earlier finite time observer designs. We also provide analogs for cases where the measurements are only available at discrete times, where we prove exponential input-to-state stability. We illustrate the advantages of our new observers using a DC motor dynamics.
\end{abstract}

Index Terms-Observer, nonlinear, robust

\section{INTRODUCTION}

$\mathbf{F}$ INITE and fixed time observers present an obvious advantage by providing estimates of the state variables of systems in finite time [1]. Fixed time observers are special cases of finite time observers where the finite convergence time is independent of the initial state. Several types of fixed time observers are available. Some use discontinuous dynamic extensions [2], time-varying high gains [3], delays, or homogeneity conditions [4].

In earlier works, e.g., [5], [6], and [7], fixed time observers are designed using dynamic extensions and a delay $\tau$. The designs rely on the invertibility of a matrix which can be problematic because it is not invertible for all $\tau$ 's and because, when it exists, the inverse can contain big terms when the delays are close to values where it is not invertible. We refer to such delays as artificial delays, because although they are not present in the given dynamics, they occur in the observers. The work [8] provides an exact calculation of state variables using a formula with several delays and the inversion of a matrix, which can also be problematic because it may be noninvertible for some delay values. Moreover, the observers in [5], [6], [8], and [7] are not reduced order.

To overcome these shortcomings, we revisit the problem of estimating the state variables of a system in finite time using an artificial delay. For a family of unperturbed systems that are affine in the unmeasured state, we propose a new family of observers that converge in fixed time when continuous output measurements are available. The observers only

Supported by NSF Grants 1711299 and 2009659 (Malisoff).

F. Mazenc is with Inria Saclay, L2S-CNRS-CentraleSupélec, 3 rue Joliot Curie, 91192, Gif-sur-Yvette, France (e-mail: frederic.mazenc@|2s.centralesupelec.fr)

M. Malisoff is with Department of Mathematics, Louisiana State University, Baton Rouge, LA 70803, USA (e-mail: malisoff@Isu.edu) estimate unmeasured variables, and so are reduced order. A key aspect of the observer design we propose is that it relies on the introduction of only one pointwise delay, which can be arbitrarily chosen. The delay is the fixed convergence time. We also establish a robustness result for the observers with respect to additive disturbances on the output measurement and dynamics. We then provide an analog for cases where the measurements are only available at discrete instants. In this case, the exponential convergence rate is proportional to the logarithm of the size of the largest sampling interval.

We use standard notation, where the dimensions of our Euclidean spaces are arbitrary, unless we indicate otherwise. The standard Euclidean 2-norm, and its induced matrix norm, are denoted by $|\cdot|,|\cdot|_{\infty}$ is the $\mathcal{L}_{\infty}$ sup norm, $|\cdot|_{S}$ is the essential supremum over sets $S$, and $I$ is the identity matrix.

\section{STUDIED SYSTEM}

We consider the class of continous-time systems

$$
\left\{\begin{aligned}
\dot{\chi}(t) & =M \chi(t)+\Psi(N \chi(t), t)+\delta_{1}(t) \\
Y(t) & =N \chi(t)+\delta_{2}(t)
\end{aligned}\right.
$$

where $\chi$ is valued in $\mathbb{R}^{n}$, the output $Y$ is valued in $\mathbb{R}^{q}$, the time dependence in $\Psi$ can represent the effects of a control, and the locally essentially bounded measurable functions $\delta_{1}$ and $\delta_{2}$ represent disturbances (but see Remark 3 for a method to use the uncertainties $\delta_{i}$ to incorporate the effects of nonlinearities in more general systems or nonlinearities in the measurements). The structure (1) and the main assumptions below are motivated by the facts that they hold for permanent magnet DC motors, pendulums, and dynamics for elastic membranes; see [9] and [10]. We assume that (1) is forward complete, and that $\Psi$ is locally Lipschitz. We also assume that the pair $(M, N)$ is observable and that $N$ has full rank.

From [11, pp. 304-306], we can deduce that there is a linear change of coordinates which yields the system

$$
\left\{\begin{aligned}
\dot{\xi}_{1}(t)= & A_{1} \xi_{1}(t)+F_{1}\left(Y(t)-\delta_{2}(t), t\right)+\epsilon_{1}(t) \\
\dot{\xi}_{2}(t)= & A_{2} \xi_{1}(t)-k \xi_{2}(t)+F_{2}\left(Y(t)-\delta_{2}(t), t\right) \\
& +\epsilon_{2}(t) \\
Y(t)= & \xi_{2}(t)+\delta_{2}(t)
\end{aligned}\right.
$$

which is affine in the unmeasured variable $\xi_{1}$, where $\xi_{1}$ is valued in $\mathbb{R}^{n-q}, \xi_{2}$ is valued in $\mathbb{R}^{q}, A_{1} \in \mathbb{R}^{(n-q) \times(n-q)}$, $A_{2} \in \mathbb{R}^{q \times(n-q)}$, the pair $\left(A_{1}, A_{2}\right)$ is observable, and $k>0$ is a constant such that $A_{1}+k I$ is invertible. Then $F_{1}$ and $F_{2}$ are locally Lipschitz, and the measurable locally essentially bounded functions $\epsilon_{i}$ represent disturbances. Although the $-k \xi_{2}(t)$ term can be incorporated into the function $F_{2}$ in (2), we keep it separate to facilitate the analysis that follows, and we write $\xi_{2}(\mathrm{t})$ as $Y(t)-\delta_{2}(t)$ in the $F_{i}$ 's in (2) to facilitate our study of the key special case where $\delta_{2}$ is the zero function. 
Changing the parameter $k$ can be done by changing $F_{2}$. One can always choose it such that $A_{1}+k I$ is invertible, by taking $k$ larger than the spectral radius of $A_{1}$.

\section{Continuous Measurement Cases}

\section{A. Assumptions and Statement of Theorem}

We construct an fixed time observer for (2), assuming:

Assumption 1: Either (i) there are two constants $K_{1} \geq 0$ and $K_{2} \geq 0$ such that

$$
\left|F_{i}(a, t)-F_{i}(b, t)\right| \leq K_{i}|a-b| \text { for } i=1,2
$$

for all $t \geq 0$ and $a$ and $b$ in $\mathbb{R}^{q}$ or (ii) $\delta_{2}(t)=0$ for all $t \geq 0$.

Let us introduce any positive constant $\tau$ and the function $\lambda: \mathbb{R} \rightarrow \mathbb{R}^{q \times(n-q)}$ defined by

$$
\lambda(r)=A_{2}\left(A_{1}+k I\right)^{-1}\left[I-e^{\left(A_{1}+k I\right) r}\right]
$$

which is well-defined because we choose $k>0$ such that the matrix $A_{1}+k I$ is invertible. We also use the matrix

$$
\mathcal{S}=\int_{-\tau}^{0} \lambda(m)^{\top} \lambda(m) \mathrm{d} m \in \mathbb{R}^{(n-q) \times(n-q)} .
$$

In the appendix below, we prove that since the pair $\left(A_{1}, A_{2}\right)$ is observable, $\mathcal{S}$ is invertible. Then we define the matrices

$$
\begin{aligned}
& \mathcal{N}=\int_{-\tau}^{0} \lambda(m)^{\top} \mathrm{d} m \in \mathbb{R}^{(n-q) \times q}, \\
& \mathcal{R}=\mathcal{S}^{-1} \mathcal{N} \in \mathbb{R}^{(n-q) \times q}, \text { and } \\
& \mathcal{H}=\left(\left(A_{1}+k I\right)^{-1}\right)^{\top} A_{2}^{\top} \in \mathbb{R}^{(n-q) \times q}
\end{aligned}
$$

and we introduce the dynamic extension

$$
\left\{\begin{array}{l}
\dot{\hat{\xi}}_{1}(t)=A_{1} \hat{\xi}_{1}(t)+F_{1}(Y(t), t) \\
\dot{\hat{\xi}}_{2}(t)=A_{2} \hat{\xi}_{1}(t)-k \hat{\xi}_{2}(t)+F_{2}(Y(t), t) \\
\dot{\psi}_{1}(t)=-k \psi_{1}(t)+\mathcal{H}\left[Y(t)-\hat{\xi}_{2}(t)\right] \\
\dot{\psi}_{2}(t)=-\left(A_{1}^{\top}+2 k I\right) \psi_{2}(t)+\mathcal{H}\left[Y(t)-\hat{\xi}_{2}(t)\right]
\end{array}\right.
$$

where $\hat{\xi}_{1}$ is valued in $\mathbb{R}^{n-q}, \hat{\xi}_{2}$ is valued in $\mathbb{R}^{q}$, and $\psi_{1}$ and $\psi_{2}$ are valued in $\mathbb{R}^{n-q}$. Finally, in terms of the functions

$$
\begin{aligned}
& \Delta_{*}(p)=e^{A_{1} p}-e^{-k p I} \text { and } \\
& \Delta_{* *}(p, q)=\int_{p}^{q} e^{A_{1}(p-\ell)} \epsilon_{1}(\ell) \mathrm{d} \ell
\end{aligned}
$$

where $k$ is from (2), we let $\epsilon_{\ddagger}$ be the $\mathbb{R}^{n-q}$-valued function

$$
\begin{aligned}
& \epsilon_{\ddagger}(t)=\mathcal{S}^{-1} \int_{t-\tau}^{t} \lambda(s-t)^{\top} \mathcal{H}^{\top} \Delta_{*}(t-s) \Delta_{* *}(s, t) \mathrm{d} s \\
& -\mathcal{S}^{-1} \int_{t-\tau}^{t} \lambda(s-t)^{\top}\left[-\int_{s}^{t} e^{k(m-t)} A_{2} \Delta_{* *}(m, s) \mathrm{d} m\right. \\
& \left.+\int_{s}^{t} e^{k(\ell-t)} \epsilon_{2}(\ell) \mathrm{d} \ell\right] \mathrm{d} s .
\end{aligned}
$$

In terms of the preceding notation and the functions

$$
\epsilon_{i}^{\sharp}(m)=K_{i}\left|\delta_{2}(m)\right|+\left|\epsilon_{i}(m)\right|
$$

for $i=1,2$ and the constants

$$
\begin{aligned}
& \bar{S}=\left|\mathcal{S}^{-1}\right| \text { and } c_{\triangle}(\tau)=\tau \bar{S}\left|A_{2}\right| e^{\left|A_{1}\right| \tau} \\
& +\bar{S}\left|A_{2}\left(A_{1}+k I\right)^{-1}\right|\left[e^{\left|A_{1}\right| \tau}+1\right] e^{\left|A_{1}\right| \tau}
\end{aligned}
$$

our first theorem is then as follows:

Theorem 1: Let (2) satisfy Assumption 1. Then, with the preceding notation, when $\delta_{2}$ is the zero function, we have

$$
\xi_{1}(t)=\xi_{e}(t)+\epsilon_{\ddagger}(t) \text { for all } t \geq \tau, \text { where }
$$

$$
\begin{aligned}
\xi_{e}(t)= & \hat{\xi}_{1}(t)+\mathcal{R}\left(\xi_{2}(t)-\hat{\xi}_{2}(t)\right) \\
& +\mathcal{S}^{-1}\left[e^{-k \tau} \psi_{1}(t-\tau)-\psi_{1}(t)\right] \\
& +\mathcal{S}^{-1}\left[\psi_{2}(t)-e^{-\left(A_{1}^{\top}+2 k I\right) \tau} \psi_{2}(t-\tau)\right] .
\end{aligned}
$$

Also, if $F_{1}$ and $F_{2}$ satisfy (3) and $\delta_{2} \neq 0$, then

$$
\xi_{1}(t)=\xi_{e}(t)+\epsilon_{\star}(t)
$$

holds for all $t \geq \tau$ where $\epsilon_{\star}$ is a function such that

$$
\begin{aligned}
\left|\epsilon_{\star}(t)\right| \leq & c_{\triangle}(\tau) \int_{t-\tau}^{t}|\lambda(s-t)| \int_{s}^{t} \epsilon_{1}^{\sharp}(m) \mathrm{d} m \mathrm{~d} s \\
& +\bar{S} \int_{t-\tau}^{t}|\lambda(s-t)| \int_{s}^{t} \epsilon_{2}^{\sharp}(m) \mathrm{d} m \mathrm{~d} s \\
& +\bar{S}|\mathcal{H}|\left\{1+e^{\left|A_{1}^{\top}+2 k I\right| \tau}\right\} \int_{t-\tau}^{t}\left|\delta_{2}(s)\right| \mathrm{d} s
\end{aligned}
$$

for all $t \geq \tau$, and the $\epsilon_{i}^{\sharp}$, s are from (10).

Remark 1: A key feature of the observer (13) is that it incorporates only one delay $\tau$, which can be any positive value because for any $\tau>0, \mathcal{S}$ is invertible; see the appendix.

Remark 2: Since

$$
|\lambda(r)| \leq\left|A_{2}\left(A_{1}+k I\right)^{-1}\right|\left[1+e^{\left|A_{1}+k I\right||r|}\right]
$$

for all $r \in \mathbb{R}$, there are constants $c_{\natural} \geq 0$ and $c_{\diamond} \geq 0$ such that $\left|\epsilon_{\ddagger}(t)\right| \leq c_{\natural}\left|\left(\epsilon_{1}, \epsilon_{2}\right)\right|_{[t-\tau, t]}$ and $\left|\epsilon_{\star}(t)\right| \leq c_{\diamond}\left|\left(\epsilon_{1}, \epsilon_{2}, \delta_{2}\right)\right|_{[t-\tau, t]}$ for all $t \geq \tau$, namely, $c_{\natural}=\bar{S} \tau^{2} \beta_{*}\left(|\mathcal{H}|\left|\Delta_{*}\right|_{[0, \tau]} e^{\left|A_{1}\right| \tau}+\right.$ $\left.\tau\left|A_{2}\right| e^{\left|A_{1}\right| \tau}+1\right)$ and $c_{\diamond}=\tau^{2} \beta_{*}\left(c_{\Delta}(\tau)\left(K_{1}+1\right)+\bar{S}\left(K_{2}+\right.\right.$ $1))+\bar{S}|\mathcal{H}| \beta_{* *} \tau$, where $\beta_{*}$ is the right side of (16) and $\beta_{* *}$ is the quantity in curly braces in (15). We illustrate the effects of these error terms in Section V.

Remark 3: In terms of the $\delta_{i}$ 's from (1), the arguments from [11, pp.304-306] imply that the $\epsilon_{i}$ 's in (2) are $\epsilon_{1}=P \delta_{1}$ and $\epsilon_{2}=N \delta_{1}$, where the matrix $P$ is chosen such that $\left[P^{\top}, N^{\top}\right]^{\top}$ is invertible, and then $\xi=\left[P^{\top}, N^{\top}\right]^{\top} \chi$. The $\delta_{i}$ 's can be used to represent the effects of unmodeled nonlinearities in the dynamics or the measurements (by letting the $\delta_{i}$ 's be the remainder terms in the Taylor approximations). This allows us to incorporate the effects of the nonlinearities in the observer error terms $\epsilon_{\ddagger}$ and $\epsilon_{*}$ from Theorem 1 .

\section{B. Proof of Theorem 1}

We introduce the variables $y=Y-\hat{\xi}_{2}$ and

$$
\begin{aligned}
& \Delta_{i}(t)=F_{i}\left(Y(t)-\delta_{2}(t), t\right)-F_{i}(Y(t), t) \text { and } \\
& x_{i}(t)=\xi_{i}(t)-\hat{\xi}_{i}(t) \text { for } i=1,2 .
\end{aligned}
$$

Then simple calculations based on (2) and (7) give

$$
\left\{\begin{aligned}
\dot{x}_{1}(t) & =A_{1} x_{1}(t)+\Delta_{1}(t)+\epsilon_{1}(t) \\
\dot{x}_{2}(t) & =A_{2} x_{1}(t)-k x_{2}(t)+\Delta_{2}(t)+\epsilon_{2}(t) \\
y(t) & =x_{2}(t)+\delta_{2}(t) .
\end{aligned}\right.
$$

Here and in the sequel, all equalities and inequalities hold for all $t \geq 0$, unless otherwise indicated.

By applying variation of parameters to (18), we obtain

$$
\begin{aligned}
& x_{1}(t)=e^{A_{1}(t-s)} x_{1}(s) \\
& +\int_{s}^{t} e^{A_{1}(t-m)}\left[\Delta_{1}(m)+\epsilon_{1}(m)\right] \mathrm{d} m
\end{aligned}
$$

and $x_{2}(t)-e^{-k(t-s)} x_{2}(s)=\rho_{1}(t, s)$

$$
+A_{2}\left(A_{1}+k I\right)^{-1}\left[e^{A_{1}(t-s)}-e^{-k(t-s) I}\right] x_{1}(s)
$$


for all $s \geq 0$ and $t \geq s$, where

$$
\begin{aligned}
& \rho_{1}(t, s)=\int_{s}^{t} e^{k(\ell-t)}\left[\Delta_{2}(\ell)+\epsilon_{2}(\ell)\right] \mathrm{d} \ell \\
& +\int_{s}^{t} e^{k(m-t)} A_{2} \int_{s}^{m} e^{A_{1}(m-\ell)}\left[\Delta_{1}(\ell)+\epsilon_{1}(\ell)\right] \mathrm{d} \ell \mathrm{d} m,
\end{aligned}
$$

and where we used the fact that

$$
\begin{aligned}
& \int_{s}^{t} e^{k(m-t)} A_{2} e^{A_{1}(m-s)} x_{1}(s) \mathrm{d} m \\
& =e^{k(s-t)} A_{2} \int_{s}^{t} e^{\left(A_{1}+k I\right)(m-s)} \mathrm{d} m x_{1}(s) \\
& =e^{k(s-t)} A_{2}\left(A_{1}+k I\right)^{-1}\left(e^{\left(A_{1}+k I\right)(t-s)}-I\right) x_{1}(s),
\end{aligned}
$$

where the $e^{k(s-t)}$ in (22) occurs because of the relation $e^{k(m-t)} A_{2} e^{A_{1}(m-s)}=e^{k(m-t)} A_{2} e^{\left(A_{1}+k I\right)(m-s)} e^{k(s-m)}=$ $e^{k(s-t)} A_{2} e^{\left(A_{1}+k I\right)(m-s)}$.

According to (19), we have

$$
\begin{aligned}
& x_{1}(s)= \\
& e^{A_{1}(s-t)} x_{1}(t)-\int_{s}^{t} e^{A_{1}(s-m)}\left[\Delta_{1}(m)+\epsilon_{1}(m)\right] \mathrm{d} m,
\end{aligned}
$$

and our formulas (4) and (8) give $\lambda(s-t)=\mathcal{H}^{\top} \Delta_{*}(t-$ $s) e^{A_{1}(s-t)}$. Hence, we can substitute (23) into (20) to obtain

$$
\lambda(s-t) x_{1}(t)=x_{2}(t)-e^{-k(t-s)} x_{2}(s)+\rho_{2}(t, s)
$$

where $\rho_{2}(t, s)=-\rho_{1}(t, s)$

$$
+\mathcal{H}^{\top} \Delta_{*}(t-s) \int_{s}^{t} e^{A_{1}(s-m)}\left[\Delta_{1}(m)+\epsilon_{1}(m)\right] \mathrm{d} m .
$$

By left multiplying both sides of (24) by $\lambda(s-t)^{\top}$, we obtain

$$
\begin{aligned}
& \lambda(s-t)^{\top} \lambda(s-t) x_{1}(t)=\lambda(s-t)^{\top} \rho_{2}(t, s) \\
& +\lambda(s-t)^{\top} x_{2}(t)-e^{-k(t-s)} \lambda(s-t)^{\top} x_{2}(s) .
\end{aligned}
$$

By integrating (26) with respect to $s$ over $[t-\tau, t]$, we obtain

$$
\begin{aligned}
& \int_{t-\tau}^{t} \lambda(s-t)^{\top} \lambda(s-t) \mathrm{d} s x_{1}(t) \\
& =\int_{t-\tau}^{t} \lambda(s-t)^{\top} \mathrm{d} s x_{2}(t) \\
& -\int_{t-\tau}^{t} e^{-k(t-s)} \lambda(s-t)^{\top} x_{2}(s) \mathrm{d} s \\
& +\int_{t-\tau}^{t} \lambda(s-t)^{\top} \rho_{2}(t, s) \mathrm{d} s \text { for all } t \geq \tau .
\end{aligned}
$$

Hence, our choices in (5)-(6), and the invertibility of $\mathcal{S}$, give

$$
\begin{aligned}
x_{1}(t)= & \mathcal{R} x_{2}(t)+\mathcal{S}^{-1} \int_{t-\tau}^{t} \lambda(s-t)^{\top} \rho_{2}(t, s) \mathrm{d} s \\
& -\mathcal{S}^{-1} \int_{t-\tau}^{t} e^{-k(t-s)} \lambda(s-t)^{\top} x_{2}(s) \mathrm{d} s .
\end{aligned}
$$

Using the formula for $\lambda$ from (4), we obtain

$$
\begin{aligned}
& x_{1}(t)=\mathcal{R} x_{2}(t)+\mathcal{S}^{-1} \int_{t-\tau}^{t} \lambda(s-t)^{\top} \rho_{2}(t, s) \mathrm{d} s \\
& -\mathcal{S}^{-1} \int_{t-\tau}^{t} e^{-k(t-s)}\left[I-e^{\left(A_{1}^{\top}+k I\right)(s-t)}\right] \mathcal{H} x_{2}(s) \mathrm{d} s
\end{aligned}
$$

with $\mathcal{H}$ defined in (6). This equality can be rewritten as

$$
\begin{aligned}
x_{1}(t)= & \mathcal{R} x_{2}(t)+\mathcal{S}^{-1} \int_{t-\tau}^{t} \lambda(s-t)^{\top} \rho_{2}(t, s) \mathrm{d} s \\
& -\mathcal{S}^{-1} \int_{t-\tau}^{t} e^{-k(t-s)} \mathcal{H} x_{2}(s) \mathrm{d} s \\
& +\mathcal{S}^{-1} \int_{t-\tau}^{t} e^{-\left(A_{1}^{\top}+2 k I\right)(t-s)} \mathcal{H} x_{2}(s) \mathrm{d} s .
\end{aligned}
$$

Since $\xi_{2}(s)=Y(s)-\delta_{2}(s)$, we have $Y-\hat{\xi}_{2}=x_{2}+\delta_{2}$, so we deduce from (7) and (30) that

$$
\begin{aligned}
& x_{1}(t)=\mathcal{R} x_{2}(t)-\mathcal{S}^{-1}\left[\psi_{1}(t)-e^{-k \tau} \psi_{1}(t-\tau)\right] \\
& +\mathcal{S}^{-1}\left[\psi_{2}(t)-e^{-\left(A_{1}^{\top}+2 k I\right) \tau} \psi_{2}(t-\tau)\right]+\epsilon_{\star}(t),
\end{aligned}
$$

where $\epsilon_{\star}(t)=\mathcal{S}^{-1}\left[\int_{t-\tau}^{t} \lambda(s-t)^{\top} \rho_{2}(t, s) \mathrm{d} s\right.$

$$
\begin{aligned}
& +\int_{t-\tau}^{t} e^{-k(t-s)} \mathcal{H} \delta_{2}(s) \mathrm{d} s \\
& \left..-\int_{t-\tau}^{t} e^{-\left(A_{1}^{\top}+2 k I\right)(t-s)} \mathcal{H} \delta_{2}(s) \mathrm{d} s\right] .
\end{aligned}
$$

Hence, (13) and (17) give $\xi_{1}=\xi_{e}+\epsilon_{\star}$ for all $t \geq \tau$.

Recalling the formula for $\rho_{2}$ in (25) and (17) and our Lipshitz condition (3) on the $F_{i}$ 's, it follows that (32) satisfies

$$
\begin{aligned}
\left|\epsilon_{\star}(t)\right| \leq & \bar{S} \int_{t-\tau}^{t}|\lambda(s-t)| \rho_{1}(t, s) \mid \mathrm{d} s \\
& +\bar{S} \int_{t-\tau}^{t}|\lambda(s-t)|\left|\mathcal{H}^{\top}\right| J_{1}(t, s) \mathrm{d} s \\
& +\bar{S}|\mathcal{H}| \int_{t-\tau}^{t} e^{-k(t-s)}\left|\delta_{2}(s)\right| \mathrm{d} s \\
& +\bar{S}|\mathcal{H}| \int_{t-\tau}^{t} e^{\left|A_{1}^{\top}+2 k I\right|(t-s)}\left|\delta_{2}(s)\right| \mathrm{d} s \\
\leq & \bar{S} \int_{t-\tau}^{t}|\lambda(s-t)| \rho_{1}(t, s) \mid \mathrm{d} s \\
& +\bar{S} \int_{t-\tau}^{t}|\lambda(s-t)|\left|\mathcal{H}^{\top}\right| J_{2}(t, s) \mathrm{d} s \\
& +\bar{S}|\mathcal{H}| \int_{t-\tau}^{t} J_{3}(t-s)\left|\delta_{2}(s)\right| \mathrm{d} s, \text { where }
\end{aligned}
$$

$$
\begin{aligned}
& J_{1}(t, s) \\
& =\left|\Delta_{*}(t-s)\right| \int_{s}^{t} e^{\left|A_{1}\right|(m-s)}\left[\left|\Delta_{1}(m)\right|+\left|\epsilon_{1}(m)\right|\right] \mathrm{d} m
\end{aligned}
$$

and the function $\Delta_{*}$ was defined in (8), and where

$$
\begin{aligned}
& J_{2}(t, s)= \\
& {\left[e^{\left|A_{1}\right| \tau}+1\right] \int_{s}^{t} e^{\left|A_{1}\right|(m-s)}\left[K_{1}\left|\delta_{2}(m)\right|+\left|\epsilon_{1}(m)\right|\right] \mathrm{d} m}
\end{aligned}
$$

and $J_{3}(r)=e^{-k r}+e^{\left|A_{1}^{\top}+2 k I\right| r}$, and where $\bar{S}$ is defined in (11). Also, when $\delta_{2}=0$, we can use (17) to get $\Delta_{1}=\Delta_{2}=0$, so our formula (9) for $\epsilon_{\ddagger}$ and (21) and (25) give $\epsilon_{\star}=\epsilon_{\ddagger}$ when $\delta_{2}=0$. Hence, since the right side of (15) is an upper bound for the right side of (33), this allows us to conclude that (15) holds for all $t \geq \tau$.

\section{Discrete Measurements Cases}

\section{A. Assumptions and Statement of Theorem}

While the observer from Section III enjoys fixed time convergence and robustness properties, it requires continuous measurements of the output, which might not always be available in practice. Therefore, we next consider cases where the variables are only measured at discrete instants.

Let $t_{j}$ be a sequence such that $t_{0}=0$ and such that there are two constants $\underline{T}>0$ and $\bar{T}>\underline{T}$ such that

$$
\underline{T} \leq t_{j+1}-t_{j} \leq \bar{T} \text { for all } j \geq 0 .
$$

We continue the notation from Section III except we consider

$$
\left\{\begin{aligned}
\dot{\xi}_{1}(t) & =A_{1} \xi_{1}(t)+F_{1}\left(\xi_{2}(t), t\right)+\epsilon_{1}(t) \\
\dot{\xi}_{2}(t) & =A_{2} \xi_{1}(t)-k \xi_{2}(t)+F_{2}\left(\xi_{2}(t), t\right)+\epsilon_{2}(t) \\
Y\left(t_{j}\right) & =\xi_{2}\left(t_{j}\right)+\delta_{2}\left(t_{j}\right) \text { for all } j \geq 0,
\end{aligned}\right.
$$

under the assumption that $F_{1}$ and $F_{2}$ satisfy (3) and where $k$ is selected as in Section II. We also use these constants:

$$
\begin{aligned}
& \varsigma_{1}=\frac{\bar{S}\left|A_{2}\right| e^{\left|A_{1}\right| \tau}}{k^{2}} \int_{-\tau}^{0}|\lambda(s)|\left(e^{k s}-s k-1\right) \mathrm{d} s \\
& \varsigma_{2}=\overline{\bar{S}} \int_{-\tau}^{0}|\lambda(s)|\left(1-e^{k s}\right) \mathrm{d} s \\
& \varsigma_{3}=\bar{S} \tau e^{\left|A_{1}\right| \tau}\left|\mathcal{H}^{\top}\right| \int_{-\tau}^{0}|\lambda(s)|\left(e^{\left|A_{1}\right| \tau}+e^{-k s}\right) \mathrm{d} s \\
& \varsigma_{4}=\bar{S}|\mathcal{H}|\left(\frac{1}{k}\left(1-e^{-k \tau}\right)+\tau e^{\left|A_{1}^{\top}+2 k I\right| \tau}\right)
\end{aligned}
$$


where $\mathcal{H}$ is from (6) as before, and $\tau$ satisfies the requirements from Section II. We introduce the dynamic extension

$$
\left\{\begin{aligned}
& \dot{\hat{\xi}}_{1}(t)=A_{1} \hat{\xi}_{1}(t)+F_{1}(\omega(t), t) \\
& \dot{\hat{\xi}}_{2}(t)=A_{2} \hat{\xi}_{1}(t)-k \hat{\xi}_{2}(t)+F_{2}(\omega(t), t) \\
& \dot{\psi}_{1}(t)=-k \psi_{1}(t)+\mathcal{H}\left[\omega(t)-\hat{\xi}_{2}(t)\right] \\
& \dot{\psi}_{2}(t)=-\left(A_{1}^{\top}+2 k I\right) \psi_{2}(t)+\mathcal{H}\left[\omega(t)-\hat{\xi}_{2}(t)\right] \\
& \dot{\omega}(t)=A_{2} \xi_{e}(t)-k \omega(t)+F_{2}(\omega(t), t) \\
& \text { for all } t \in\left[t_{j}, t_{j+1}\right) \text { and } j \geq 0 \\
& \omega\left(t_{j}\right)= Y\left(t_{j}\right) \text { for all } j \geq 0
\end{aligned}\right.
$$

where $\xi_{e}$ is defined as in (13). We prove:

Theorem 2: Let the constant $\bar{T}$ in (36) be such that

$$
\bar{T} \mu<1, \text { where }
$$

$$
\mu=\left|A_{2}\right| q_{1}+k+K_{2} \text { and } q_{1}=\left(\varsigma_{1}+\varsigma_{3}\right) K_{1}+\varsigma_{2} K_{2}+\varsigma_{4}
$$

using the constants (38). Then we can find positive constants $a_{1}$ and $a_{2}$ such that all solutions of (37) and (39) satisfy

$$
\begin{aligned}
& \left|\xi_{1}(t)-\xi_{e}(t)\right| \leq a_{1}\left|\xi_{2}-\omega\right|_{[r-2 \tau-\bar{T}, r]} e^{\frac{\ln (\bar{T} \mu)}{\tau+\bar{T}}(t-r)} \\
& \quad+a_{2} \sup _{\ell \in[r-\bar{T}-2 \tau, t]}\left[\left|\epsilon_{1}(\ell)\right|+\left|\epsilon_{2}(\ell)\right|+\left|\delta_{2}(\ell)\right|\right]
\end{aligned}
$$

for all $r \geq 2 \tau+\bar{T}$ and $t \geq r+\tau$.

Remark 4: The inequality (42) is of ISS type because

$$
\frac{\ln (\bar{T} \mu)}{\tau+\bar{T}}<0
$$

by (40). Moreover, since $\mu$ is independent of $\bar{T}$, the left side of (43) converges to $-\infty$ as $\bar{T} \rightarrow 0^{+}$. Therefore, we can have arbitrarily large rates of convergence of the estimation error $\left|\xi_{1}(t)-\xi_{e}(t)\right|$ to 0 when the $\epsilon_{i}$ 's are zero, by choosing the sample times $t_{i}$ such that $\bar{T}$ is small enough. In practice, this faster sampling can often be achieved by upgrading to a faster digital signal processor (or DSP) in a lab. Our proof of Theorem 2 can be used to easily get formulas for the $a_{i}$ 's in (42). For example, our proof shows that we can choose $a_{1}=$ $q_{1} e^{-R_{*} \tau}$ and $a_{2}=q_{2}+q_{1} \bar{T}^{\sharp} /(1-\bar{T} \mu)$, where $R_{*}$ is the left side of (43), $\bar{T}^{\sharp}=\bar{T}\left(\left|A_{2}\right| q_{2}+1\right)+1$ and $q_{2}=\max \left\{\varsigma_{1}+\varsigma_{3}, \varsigma_{2}\right\}$. Also, since the $F_{i}$ 's are known, we can readily compute the Lipschitz constants $K_{i}$ that are needed to compute $\mu$.

\section{B. Proof of Theorem 2}

Our proof will use the variables

$$
\begin{aligned}
& \tilde{\omega}(t)=\xi_{2}(t)-\omega(t), \text { and } x_{i}(t)=\xi_{i}(t)-\hat{\xi}_{i}(t) \\
& \text { and } \kappa_{i}(t)=F_{i}\left(\xi_{2}(t), t\right)-F_{i}(\omega(t), t) \text { for } i=1,2 .
\end{aligned}
$$

Then $\omega-\hat{\xi}_{2}=\xi_{2}-\tilde{\omega}-\hat{\xi}_{2}=x_{2}-\tilde{\omega}$, so we obtain

$$
\left\{\begin{aligned}
\dot{x}_{1}(t)= & A_{1} x_{1}(t)+\kappa_{1}(t)+\epsilon_{1}(t) \\
\dot{x}_{2}(t)= & A_{2} x_{1}(t)-k x_{2}(t)+\kappa_{2}(t)+\epsilon_{2}(t) \\
\dot{\psi}_{1}(t)= & -k \psi_{1}(t)+\mathcal{H} x_{2}(t)-\mathcal{H} \tilde{\omega}(t) \\
\dot{\psi}_{2}(t)= & -\left(A_{1}^{\top}+2 k I\right) \psi_{2}(t)+\mathcal{H} x_{2}(t)-\mathcal{H} \tilde{\omega}(t) \\
\dot{\tilde{\omega}}(t)= & A_{2}\left[\xi_{1}(t)-\xi_{e}(t)\right]-k \tilde{\omega}(t)+\kappa_{2}(t)+\epsilon_{2}(t) \\
& \text { for all } t \in\left[t_{j}, t_{j+1}\right) \text { and } j \geq 0 \\
\tilde{\omega}\left(t_{j}\right)= & -\delta_{2}\left(t_{j}\right) \text { for all } j \geq 0 .
\end{aligned}\right.
$$

We also use the $\mathbb{R}^{n-q}$-valued variables

$$
\begin{gathered}
\gamma_{1}(t)=-\mathcal{S}^{-1} \int_{t-\tau}^{t} \lambda(s-t)^{\top} \mathcal{H}^{\top} \Delta_{*}(t-s) J_{a}(t, s) \mathrm{d} s \\
-\mathcal{S}^{-1} \int_{t-\tau}^{t} \lambda(s-t)^{\top} \int_{s}^{t} e^{k(m-t)} A_{2} J_{a}(s, m) \mathrm{d} m \mathrm{~d} s \\
-\mathcal{S}^{-1} \int_{t-\tau}^{t} \lambda(s-t)^{\top} \int_{s}^{t} e^{k(\ell-t)}\left[\kappa_{2}(\ell)+\epsilon_{2}(\ell)\right] \mathrm{d} \ell \mathrm{d} s, \\
\gamma_{2}(t)=-\mathcal{S}^{-1} \int_{t-\tau}^{t} e^{-k(t-s)} \mathcal{H} \tilde{\omega}(s) \mathrm{d} s \\
+\mathcal{S}^{-1} \int_{t-\tau}^{t} e^{\left(A_{1}^{\top}+2 k I\right)(-t+s)} \mathcal{H} \tilde{\omega}(s) \mathrm{d} s,
\end{gathered}
$$

and

$$
\begin{aligned}
x_{a}(t)= & \mathcal{R} x_{2}(t)-\mathcal{S}^{-1}\left[\psi_{1}(t)-e^{-k \tau} \psi_{1}(t-\tau)\right] \\
& +\mathcal{S}^{-1}\left[\psi_{2}(t)-e^{-\left(A_{1}^{\top}+2 k I\right) \tau} \psi_{2}(t-\tau)\right],
\end{aligned}
$$

where $J_{a}(s, m)=\int_{s}^{m} e^{A_{1}(m-\ell)}\left[\kappa_{1}(\ell)+\epsilon_{1}(\ell)\right] \mathrm{d} \ell$.

The rest of the proof of the theorem consists of two steps. In the first step, we prove that the preceding variables satisfy

$$
\xi_{1}(t)=\xi_{e}(t)+\gamma_{1}(t)+\gamma_{2}(t) \text { for all } t \geq \tau .
$$

Then, we bound $\gamma_{1}(t)+\gamma_{2}(t)$ by the right side of (42).

First Step. Since the $\left(x_{1}, x_{2}\right)$-dynamics of (45) agree with the first two equations of (18) except with the $\Delta_{i}$ 's replaced by the $\kappa_{i}$ 's, the same reasoning that led to (30) gives

$$
\begin{aligned}
& x_{1}(t)=\mathcal{R} x_{2}(t)-\mathcal{S}^{-1} \int_{t-\tau}^{t} e^{-k(t-s)} \mathcal{H} x_{2}(s) \mathrm{d} s \\
& +\mathcal{S}^{-1} \int_{t-\tau}^{t} e^{-\left(A_{1}^{\top}+2 k I\right)(t-s)} \mathcal{H} x_{2}(s) \mathrm{d} s+\gamma_{1}(t) .
\end{aligned}
$$

Also, by applying the method of variation of parameters separately to the $\psi_{1}$ and $\psi_{2}$ dynamics in (45), we obtain

$$
\begin{aligned}
& \int_{t-\tau}^{t} e^{-k(t-s)} \mathcal{H} x_{2}(s) \mathrm{d} s= \\
& \psi_{1}(t)-e^{-k \tau} \psi_{1}(t-\tau)+\int_{t-\tau}^{t} e^{-k(t-s)} \mathcal{H} \tilde{\omega}(s) \mathrm{d} s
\end{aligned}
$$

and

$$
\begin{aligned}
& \int_{t-\tau}^{t} e^{-\left(A_{1}^{\top}+2 k I\right)(t-s)} \mathcal{H} x_{2}(s) \mathrm{d} s= \\
& \psi_{2}(t)-e^{-\left(A_{1}^{\top}+2 k I\right) \tau} \psi_{2}(t-\tau) \\
& +\int_{t-\tau}^{t} e^{\left(A_{1}^{\top}+2 k I\right)(-t+s)} \mathcal{H} \tilde{\omega}(s) \mathrm{d} s
\end{aligned}
$$

for all $t \geq \tau$. By combining (51)-(53), we obtain

$$
\begin{aligned}
x_{1}(t)= & \mathcal{R} x_{2}(t)-\mathcal{S}^{-1}\left[\psi_{1}(t)-e^{-k \tau} \psi_{1}(t-\tau)\right. \\
& \left.+\int_{t-\tau}^{t} e^{-k(t-s)} \mathcal{H} \tilde{\omega}(s) \mathrm{d} s\right]+\gamma_{1}(t) \\
& +\mathcal{S}^{-1}\left[\psi_{2}(t)-e^{-\left(A_{1}^{\top}+2 k I\right) \tau} \psi_{2}(t-\tau)\right. \\
& \left.+\int_{t-\tau}^{t} e^{\left(A_{1}^{\top}+2 k I\right)(-t+s)} \mathcal{H} \tilde{\omega}(s) \mathrm{d} s\right]
\end{aligned}
$$

for all $t \geq \tau$ Hence, our choices (46)-(48), and our choice of $x_{1}$ in (44) and our formula (13) for $\xi_{e}$, give $x_{1}=x_{a}+\gamma_{1}+\gamma_{2}$ and $x_{1}-x_{a}=\xi_{1}-\xi_{e}$, which we can combine to obtain (50).

Second Step. From (50), it follows that

$$
\begin{aligned}
& \left|\xi_{1}(t)-\xi_{e}(t)\right| \leq \\
& \bar{S} \int_{t-\tau}^{t}|\lambda(s-t)| \int_{s}^{t}\left|A_{2}\right| e^{k(m-t)} J_{4}(s, m) \mathrm{d} m \mathrm{~d} s \\
& +\bar{S} \int_{t-\tau}^{t}|\lambda(s-t)| \int_{s}^{t} e^{k(\ell-t)}\left[\left|\kappa_{2}(\ell)\right|+\left|\epsilon_{2}(\ell)\right|\right] \mathrm{d} \ell \mathrm{d} s \\
& +\bar{S} \int_{t-\tau}^{t}|\lambda(s-t)|\left|\mathcal{H}^{\top}\right|\left[e^{\left|A_{1}\right| \tau}+e^{k(s-t)}\right] J_{4}(s, t) \mathrm{d} s \\
& +\bar{S} \int_{t-\tau}^{t}\left(e^{k(s-t)}+e^{\left|A_{1}^{\top}+2 k I\right| \tau}\right)|\mathcal{H}||\tilde{\omega}(s)| \mathrm{d} s
\end{aligned}
$$


for all $t \geq \tau$, where

$$
J_{4}(s, m)=\int_{s}^{m} e^{\left|A_{1}\right| \tau}\left[\left|\kappa_{1}(\ell)\right|+\left|\epsilon_{1}(\ell)\right|\right] \mathrm{d} \ell .
$$

Moreover, our choices of the $\kappa_{i}$ 's in (44) and (3) give $\left|\kappa_{2}(\ell)\right| \leq K_{2}|\tilde{w}(\ell)|$ when $s \leq \ell \leq t$, and so also

$$
J_{4}(s, m) \leq(m-s) e^{\left|A_{1}\right| \tau}\left(K_{1}|\tilde{w}|_{[s, m]}+\left|\epsilon_{1}\right|_{[s, m]}\right)
$$

when $s \leq m \leq t$. Therefore, by upper bounding the right side of (55) and then collecting coefficients of $|\tilde{w}|_{[t-\tau, t]}$ and $\left|\epsilon_{i}\right|_{[t-\tau, t]}$ for $i=1,2$ in the result, it follows from (38) that

$$
\begin{aligned}
& \left|\xi_{1}(t)-\xi_{e}(t)\right| \leq \\
& q_{1}|\tilde{w}|_{[t-\tau, t]}+q_{2}\left(\left|\epsilon_{1}\right|_{[t-\tau, t]}+\left|\epsilon_{2}\right|_{[t-\tau, t]}\right) \text { for all } t \geq \tau,
\end{aligned}
$$

where $q_{1}$ is from (41) and $q_{2}=\max \left\{\varsigma_{1}+\varsigma_{3}, \varsigma_{2}\right\}$.

By combining (45) and (58), and recalling (3), we get

$$
\begin{aligned}
|\dot{\tilde{\omega}}(t)| \leq & \left|A_{2}\right|\left|\xi_{1}(t)-\xi_{e}(t)\right|+k|\tilde{\omega}(t)|+\left|\epsilon_{2}(t)\right| \\
& +\left|F_{2}\left(\xi_{2}(t), t\right)-F_{2}(\omega(t), t)\right| \\
\leq & \left(\left|A_{2}\right| q_{1}+k+K_{2}\right)|\tilde{w}|_{[t-\tau, t]}+\epsilon_{£}(t)
\end{aligned}
$$

for all $t \in\left[t_{j}, t_{j+1}\right)$ and all $j \geq 0$ when $t \geq \tau$, where $\epsilon_{£}(t)=$ $\left|A_{2}\right| q_{2}\left(\left|\epsilon_{1}\right|_{[t-\tau, t]}+\left|\epsilon_{2}\right|_{[t-\tau, t]}\right)+\left|\epsilon_{2}(t)\right|$. Since

$$
\tilde{\omega}(t)=\tilde{\omega}\left(t_{j}\right)+\int_{t_{j}}^{t} \dot{\tilde{\omega}}(\ell) \mathrm{d} \ell
$$

for all $t \in\left[t_{j}, t_{j+1}\right)$, we deduce that, for all $t \geq \bar{T}+\tau$,

$$
\begin{aligned}
|\tilde{\omega}(t)| \leq & \left|\tilde{\omega}\left(t_{j}\right)\right|+\bar{T}\left(\left|A_{2}\right| q_{1}+k+K_{2}\right)|\tilde{w}|_{\mathcal{S}_{t}} \\
& +\bar{T}\left|\epsilon_{£}\right|_{[t-\bar{T}, t]} \\
\leq & \bar{T} \mu|\tilde{\omega}|_{\mathcal{S}_{t}}+\bar{T}^{\sharp}\left(\sum_{j=1}^{2}\left|\epsilon_{i}\right|_{\mathcal{S}_{t}}+\left|\delta_{2}\right|_{\mathcal{S}_{t}}\right)
\end{aligned}
$$

when $t \in\left[t_{j}, t_{j+1}\right), j \geq 0, t \geq r$, and $r \geq \bar{T}+\tau$, where the suprema are over $\mathcal{S}_{t}=[t-\bar{T}-\tau, t], \bar{T}^{\sharp}=\bar{T}\left(\left|A_{2}\right| q_{2}+1\right)+1$, the last inequality in (61) used the bound $\left|\tilde{\omega}\left(t_{j}\right)\right|=\left|\delta_{2}\left(t_{j}\right)\right|$, and $\mu$ is from (41). Using (40), it follows from applying [12, Lemma 1] to $w_{0}(t)=|\tilde{\omega}(t+r)|$ that, for all $t \geq r$,

$$
\begin{aligned}
& |\tilde{\omega}(t)| \leq|\tilde{\omega}|_{[r-\tau-\bar{T}, r]} e^{\frac{\ln (\bar{T} \mu)}{\tau+\bar{T}}(t-r)}+\mathcal{T}_{\epsilon}(t, r), \\
& \text { where } \mathcal{T}_{\epsilon}(t, r)=\frac{\bar{T}^{\sharp}}{1-\bar{T} \mu}\left(\sum_{j=1}^{2}\left|\epsilon_{i}\right|_{\mathcal{S}_{t}}+\left|\delta_{2}\right|_{\mathcal{S}_{t}}\right) .
\end{aligned}
$$

The theorem now follows by using (62) to upper bound the first right side term in (58).

\section{ILLUStrations}

Consider this model for a single-link direct-drive manipulator actuated by a permanent magnet DC brush motor [13]:

$$
\begin{aligned}
& M \ddot{q}+B \dot{q}+N \sin (q)=\mathcal{I} \text { and } \\
& L \dot{\mathcal{I}}=V_{e}-R \mathcal{I}-K_{B} \dot{q}, \\
& \text { where } M=\frac{J}{K_{\tau}}+\frac{m L_{0}^{2}}{3 K_{\tau}}+\frac{M_{0} L_{0}^{2}}{K_{\tau}}+\frac{2 M_{0} R_{0}^{2}}{5 K_{\tau}}, \\
& N=\frac{m L_{0} G}{2 K_{\tau}}+\frac{M_{0} L_{0} G}{K_{\tau}}, \text { and } B=\frac{B_{0}}{K_{\tau}}
\end{aligned}
$$

where the physical meanings of the positive constants $m, J$, $L_{0}, M_{0}, B_{0}, R_{0}, G, K_{\tau}, R, L, K_{B}$, and $V_{e}$ is explained in [13], $q(t)$ is the position of the load (which is the angular motor position), and $\mathcal{I}(t)$ is the motor armature current. We assume that perturbed measurements of $q$ are available.

The model (64) has been studied extensively. For instance, see [10] for continuous-discrete observers for (64), and [14] for full order observers with sampling and input delays. However, we believe that the problem we will solve of building reduced order observers for (64) with arbitrarily small fixed convergence times $\tau$ and a single delay was open.

By also allowing additive uncertainties in the model (64) and in the measurements, we obtain the dynamics

$$
\left\{\begin{aligned}
\dot{\chi}_{1}(t)= & \chi_{2}(t)+\delta_{1,1}(t) \\
\dot{\chi}_{2}(t)= & b_{1} \chi_{3}(t)-a_{1} \sin \left(\chi_{1}(t)\right)-a_{2} \chi_{2}(t) \\
& +\delta_{1,2}(t) \\
\dot{\chi}_{3}(t)= & b_{0} u(t)-a_{3} \chi_{2}(t)-a_{4} \chi_{3}(t)+\delta_{1,3}(t) \\
Y(t)= & \chi_{1}(t)+\delta_{2}(t)
\end{aligned}\right.
$$

where $\chi_{1}=q, \chi_{2}=\dot{q}, \chi_{3}=\mathcal{I}, a_{1}=N / M, a_{2}=B / M$, $a_{3}=K_{B} / L, a_{4}=R / L, b_{0}=1 / L$, and $b_{1}=1 / M$, and $u=V_{e}$ is the control. As in [6], we choose $b_{0}=40, b_{1}=15$, $a_{1}=35, a_{2}=1, a_{3}=36.4$ and $a_{4}=200$.

Adopting the notation $\xi_{2}=\chi_{1}, \xi_{1,1}=\chi_{2}, \xi_{1,2}=\chi_{3}$, $\varepsilon_{1,1}(t)=\delta_{1,2}(t), \varepsilon_{1,2}(t)=\delta_{1,3}(t), \varepsilon_{2}(t)=\delta_{1,1}(t)$, and $\varepsilon_{3}(t)=\delta_{2}(t)$, we can rewrite the system (65) as

$$
\left\{\begin{aligned}
\dot{\xi}_{1,1}(t)= & -a_{2} \xi_{1,1}(t)+b_{1} \xi_{1,2}(t) \\
& -a_{1} \sin \left(Y(t)-\delta_{2}(t)\right)+\varepsilon_{1,1}(t) \\
\dot{\xi}_{1,2}(t)= & -a_{3} \xi_{1,1}(t)-a_{4} \xi_{1,2}(t)+b_{0} u(t) \\
& +\varepsilon_{1,2}(t) \\
\dot{\xi}_{2}(t)= & \xi_{1,1}(t)-k \xi_{2}(t)+k\left[Y(t)-\delta_{2}(t)\right]+\varepsilon_{2}(t) \\
Y(t)= & \xi_{2}(t)+\delta_{2}(t)
\end{aligned}\right.
$$

for a constant $k>0$ that will be specified.

Then the notation of Sections II-III produces the choices

$$
A_{1}=\left[\begin{array}{cc}
-a_{2} & b_{1} \\
-a_{3} & -a_{4}
\end{array}\right], A_{2}=\left[\begin{array}{ll}
1 & 0
\end{array}\right],
$$

$F_{1}(s, t)=\left(-a_{1} \sin (s), b_{0} u(t)\right), F_{2}(s, t)=k s, K_{1}=a_{1}$ and $K_{2}=k$. With the preceding parameter choices, $A_{1}+k I$ is invertible when $k^{2}-201 k+746 \neq 0$. Thus we can take any $k>0$ that is not a root of $k^{2}-201 k+746$. The preceding choices can then be used to write the dynamic extensions from our theorems, and then Theorem 1 provides the exact value of $\xi_{1}$. The preceding observer contrasts significantly with the fixed time observer for (65) that was presented in [6, Section 5.2], whose fixed convergence time $\tau$ is required to be such that $e^{-H \tau}-e^{-\tau A}$ is invertible where $H=A+L_{A} C$ for a suitable matrix $L_{A}$, and where $C$ is from the representation $y=C x$ of the output in terms of the state $x$. Moreover, [6, Section 5.2] produces large coefficients in the final estimation error under discrete time measurements for small $\tau>0$ values. Hence, we believe that the observer designs from this work offer potential advantages over previously available observers.

We simulated the observers from Theorems 1-2 from Sections III-IV for the preceding motor model using the program Mathematica, and we report the results in Fig. 1. Fig. 2 shows the effects of changing the observer parameter $k$ (e.g., from (4)), with all other parameters kept the same as the simulations in Fig. 1. In both figures, we used the same motor model parameter values, initial states for (66), parameter $\tau=0.1$, 
and uncertainties that were used in the observers for the motor dynamics in [6, Section 5.2], which used observers from [6, Theorems 2 and 4] (which were not reduced order). We chose $\bar{T}=\underline{T}=0.1$. We plotted the first component of the observer error (for estimating $\xi_{1,1}$ ) in red, and the second observer error component (for $\xi_{1,2}$ ) in blue.

In Fig. 3 below, we also show Mathematica simulations using the observers from both theorems from [10], using the preceding parameter values, and the matrix $L=[-1,3]^{\top}$ and parameter values $\nu=0.1$ and $g=20$ from [10]. Since Figs. 1-2 show improved performance in terms of settling terms and observer errors after time $\tau=0.1$ (e.g., $\epsilon_{\ddagger}$ from Theorem 1) compared with [6] and [10] (while reducing the order of the observer compared with [6]), they help illustrate this work; see, e.g., [6, Fig. 6], where the observation errors provided by the upper or lower bound from [6, Theorem 4] were greater than 9 after 1.5 seconds when nonzero uncertainties were present, whereas our observation errors from using Theorem 2 from Section IV above stayed below 1 after 0.15 seconds.
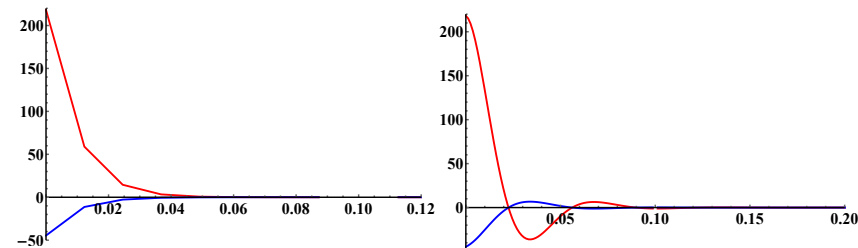

Fig. 1. Observer Error Components for (66) from Theorem 1 (Left) and Theorem 2 (Right) with $k=100$ using Parameter Values from [6].

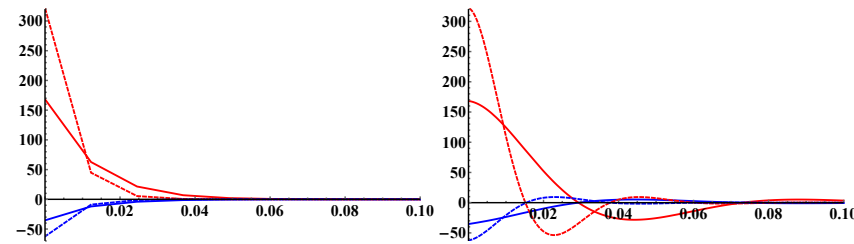

Fig. 2. Observer Error Components for (66) from Theorem 1 (Left) and Theorem 2 (Right) with $\boldsymbol{k}=\mathbf{7 5}$ (Solid) and $\boldsymbol{k}=\mathbf{1 5 0}$ (Dashed) using Parameter Values from [6].

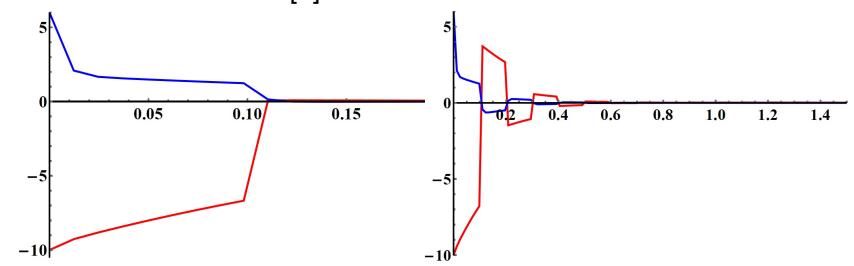

Fig. 3. Observer Error Components for (66) using Theorem 1 from [10] (Left) and Theorem 2 from [10] (Right) using Parameter Values from [6]

\section{CONClusion}

We solved significant observer design problems for systems that are linear in the unmeasured variables and with continuous but perturbed measurements, using a novel Gramian approach which eliminates an invertibility condition on the fixed convergence time from prior works such as [6], while only requiring a single delay in the observer. We also provided an analog for cases where only discrete measurements are available and where we instead get arbitrarily large exponential convergence rates. Our examples illustrated the potential advantages of our new approaches and a tradeoff between sample rates in the measurements and the parameter choices in our observer. We aim to obtain extensions for systems with state delays or measurement delays.

\section{APPENDIX: INVERTIBILITY OF THE MATRIX $\mathcal{S}$}

Let us prove that the matrix (5) is invertible, which was needed for the observer designs from our theorems. Let $V \in$ $\mathbb{R}^{n-q}$ be a vector such that $\mathcal{S} V=0$. Then

$$
\int_{-\tau}^{0} V^{\top} \lambda(m)^{\top} \lambda(m) V \mathrm{~d} m=0 .
$$

As an immediate consequence, we get $\lambda(m) V=0$ for all $m \in$ $[-\tau, 0]$. It follows that for all integers $j>0, \lambda^{(j)}(0) V=0$. Also, simple calculations give $\lambda^{(j)}(0)=-A_{2}\left(A_{1}+k I\right)^{j-1}$. Thus $A_{2}\left(A_{1}+k I\right)^{l} V=0$ for all integers $l \geq 0$. Using the fact that these equalities are equivalent to

$$
A_{2} \sum_{j=0}^{l} C_{l}^{j} A_{1}^{j} V=0
$$

for suitable nonzero integers $C_{l}^{j}$, we deduce that $A_{2} A_{1}^{l} V=0$ for all integers $l \geq 0$, by induction on $l$. Since $\left(A_{1}, A_{2}\right)$ is observable, it follows that $V=0$. This allows us to conclude.

\section{REFERENCES}

[1] F. Lopez-Ramirez, A. Polyakov, D. Efimov, and W. Perruquetti, "Finitetime and fixed-time observer design: Implicit Lyapunov function approach," Automatica, vol. 87, pp. 52-60, 2018.

[2] T. Raff and F. Allgower, "An observer that converges in finite time due to measurement-based state updates," IFAC Proceedings Volumes, vol. 41, no. 2, pp. 2693-2695, 2008.

[3] J. Holloway and M. Krstic, "Prescribed-time observers for linear systems in observer canonical form," IEEE Trans. Automat. Control, vol. 64, no. 9, pp. 3905-3912, 2019.

[4] H. Silm, R. Ushirobira, D. Efimov, J. Richard, and W. Michiels, "A note on distributed finite-time observers," IEEE Trans. Automat. Control, vol. 64, no. 2, pp. 759-766, 2019.

[5] R. Engel and G. Kreisselmeier, "A continuous time observer which converges in finite time," IEEE Trans. Automat. Control, vol. 47, no. 7, pp. 1202-1204, 2002

[6] F. Mazenc, E. Fridman, and W. Djema, "Estimation of solutions of observable nonlinear systems with disturbances," Systems and Control Letters, vol. 79, pp. 47-58, 2015.

[7] F. Sauvage, M. Guay, and D. Dochain, "Design of a nonlinear finite time converging observer for a class of nonlinear systems," Journal of Control Science and Engineering, vol. 2007, no. 36954, p. 9pp., 2007.

[8] F. Mazenc, S.-I. Niculescu, and N. Bekiaris-Liberis, "Asymptotic stabilization of linear time-varying systems with input delays via delayed static output feedback," in Proceedings of the American Control Conference, 2015, pp. 4591-4596.

[9] F. Mazenc, S. Ahmed, and M. Malisoff, "Finite time estimation through a continuous-discrete observer," International Journal of Robust and Nonlinear Control, vol. 28, no. 16, pp. 4831-4849, 2018.

[10] F. Mazenc, M. Malisoff, and Z. Jiang, "Reduced order fast converging observer for systems with discrete measurements and sensor noise," Systems and Control Letters, vol. 150, no. 104892, 2021.

[11] D. Luenberger, Introduction to Dynamic Systems. New York: John Wiley and Sons, 1979.

[12] F. Mazenc, M. Malisoff, and S.-I. Niculescu, "Stability and control design for time-varying systems with time-varying delays using a trajectory-based approach," SIAM Journal on Control and Optimization, vol. 55, no. 1, pp. 533-556, 2017.

[13] D. Dawson, J. Carroll, and M. Schneider, "Integrator backstepping control of a brush dc motor turning a robotic load," IEEE Trans. Control Syst. Tech., vol. 2, no. 3, pp. 233-244, 1994.

[14] F. Mazenc and M. Malisoff, "Continuous discrete sequential observers for time-varying systems under sampling and input delays," IEEE Trans. Automat. Control, vol. 65, no. 4, pp. 1704-1709, 2020. 